\title{
Meningioma of the Fourth Ventricle
}

\author{
-Case Report-
Shinichi NAKANo, Hisao UEHARA, Shinichiro WAKISAKA and Kazuo KINOSHITA

Department of Neurosurgery, Miyazaki Medical College, Miyazaki

\begin{abstract}
A 58-year-old female had a 1-year history of headache, ataxia, and taste disturbance and then developed speech disturbance. She was found to have a meningioma of the fourth ventricle, a very rare site for this type of tumor. It did not adhere to the cerebellar tentorium or dura mater and was totally removed without difficulty. Magnetic resonance imaging, but not computed tomography or angiography, provided adequate information for correct diagnosis. The literature concerning fourth ventricle meningiomas is reviewed and the diagnostic advantages and limitations of computed tomography, angiography, and magnetic resonance imaging are discussed.
\end{abstract}

Key words: meningioma, fourth ventricle, computed tomography, magnetic resonance imaging, angiography

\section{Introduction}

Intraventricular meningiomas usually occur in the lateral or third ventricles, and very rarely arise in the fourth ventricle. In 1938 Sachs reported the first case of meningioma of the fourth ventricle. ${ }^{3)}$ Since then, only 23 such cases have been described. ${ }^{1,2,4-9)}$ We report an additional case and stress the diagnostic value of magnetic resonance (MR) imaging.

\section{Case Report}

The patient was a 58-year-old female who complained of headache, ataxia, and speech disturbance. The ataxic gait and morning headache had begun in the summer of 1985, and in the autumn of that year she had noticed a taste disturbance. The ataxia and headache gradually worsened and in June, 1986 she developed speech disturbance. A computed tomographic (CT) scan disclosed a cerebellar tumor, and she was admitted to our department on June 27 , 1986.

Neurological examination revealed horizontal nystagmus to the left, dysarthria, right extremity ataxia, and ataxic gait. She had no papilledema. Pre-

Received November 4, 1987; Accepted July 14,

contrast CT demonstrated a high-density mass in the right cerebellar hemisphere and vermis. It markedly enhanced with contrast medium. The fourth ventricle was displaced to the left. Axial CT did not reveal whether or not the tumor was attached to the cerebellar tentorium (Fig. 1).
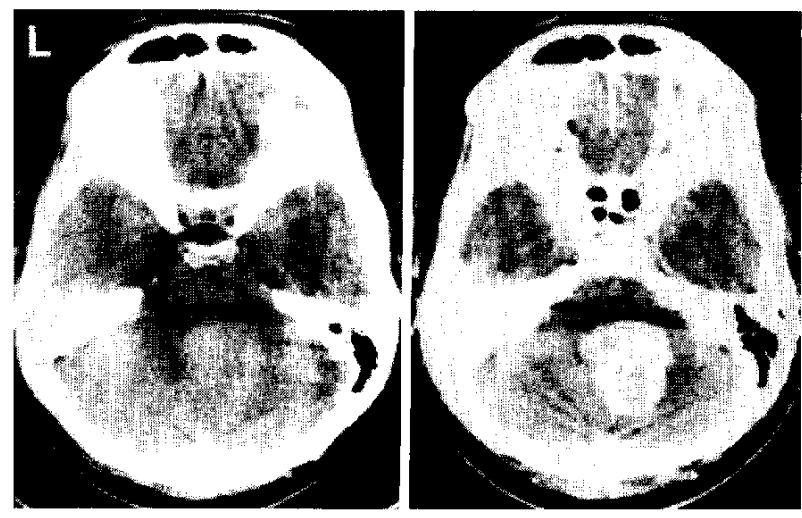

Fig. 1 Precontrast CT (left) showing a high-density mass in the right cerebellar hemisphere and vermis. It markedly enhanced with contrast medium (right). It appears well demarcated from the surrounding brain tissue, although axial CT failed to disclose whether or not the tumor adhered to the cerebellar tentorium. 


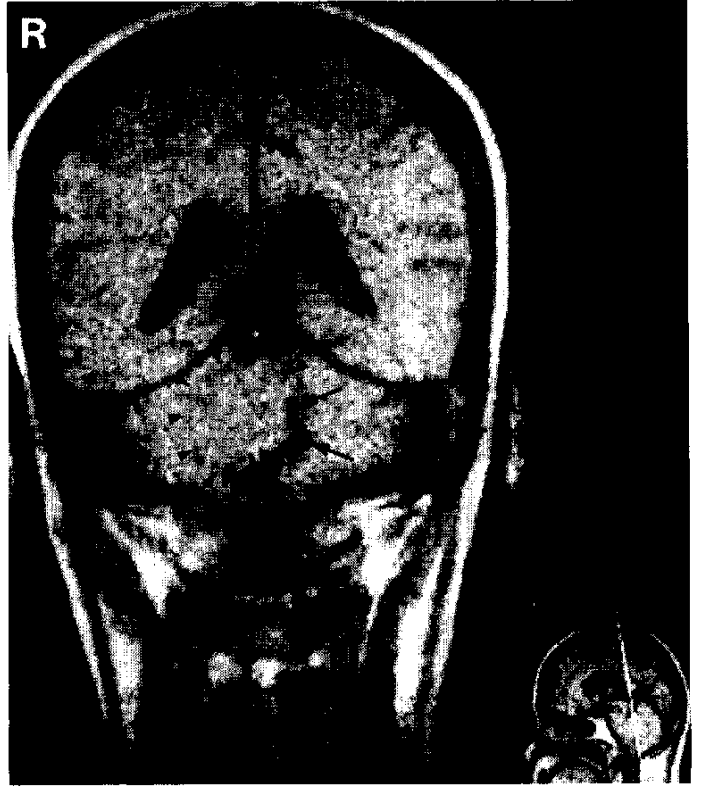

Fig. 2 A T $_{1}$-weighted coronal MR image revealing an isointense mass (arrowheads) displacing the fourth ventricle (arrows) to the left.

MR imaging was performed with a $0.1 \mathrm{~T}$ scanner. In the short spin echo (SE) images [repetition time $(\mathrm{TR})=500 \mathrm{msec}$; echo delay time $(\mathrm{TE})=30 \mathrm{msec}$, the tumor was delineated as an isointense area. A coronal short SE image showed leftward displacement of the fourth ventricle (Fig. 2). In the long SE images ( $\mathrm{TR}=1500 \mathrm{msec}$; $\mathrm{TE}=80 \mathrm{msec}$ ), it was expressed as a high-intensity area. A sagittal long SE image showed that the tumor was unattached to the cerebellar tentorium and that it displaced the brainstem anteriorly (Fig. 3). The fourth ventricle was totally occupied by the tumor, which appeared well demarcated.

Left vertebral angiography showed heterogeneous tumor staining during the capillary and venous phases. The right superior cerebellar artery seemed to be the main feeder for the tumor. The right posterior inferior cerebellar artery was not visible (Fig. 4). Right vertebral angiography was attempted but was unsuccessful owing to catheterization difficulty. External carotid angiography was not performed.

These radiological findings strongly suggested meningioma. However, because the tumor was not adherent to the cerebellar tentorium and because an abdominal echogram had disclosed multiple small cysts in the right kidney, there was also the possibility of hemangioblastoma.

A suboccipital craniectomy exposed an encapsulated tumor displacing the fourth ventricle to the

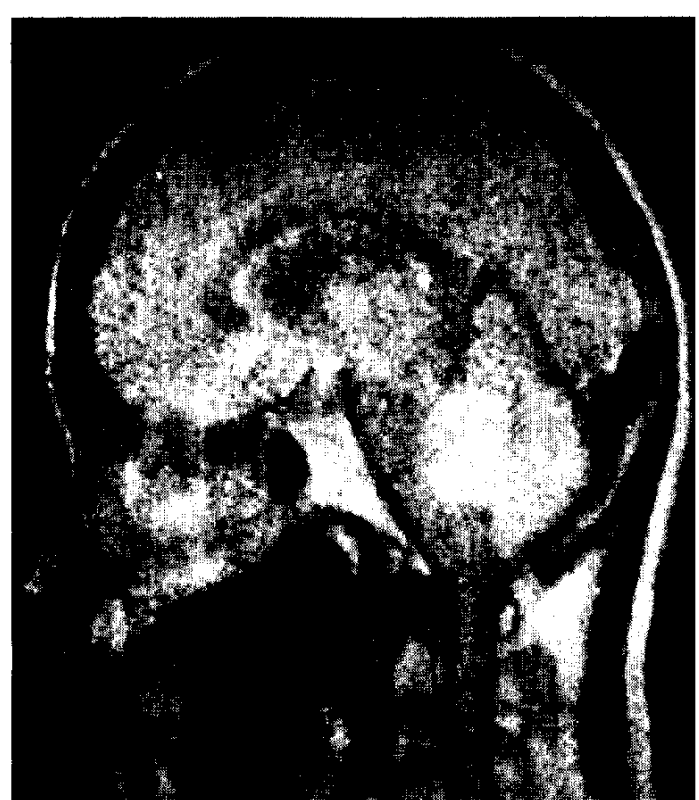

Fig. 3 A $\mathrm{T}_{2}$-weighted sagittal MR image disclosing a high-intensity mass displacing the brainstem anteriorly. It was not attached to the cerebellar tentorium.

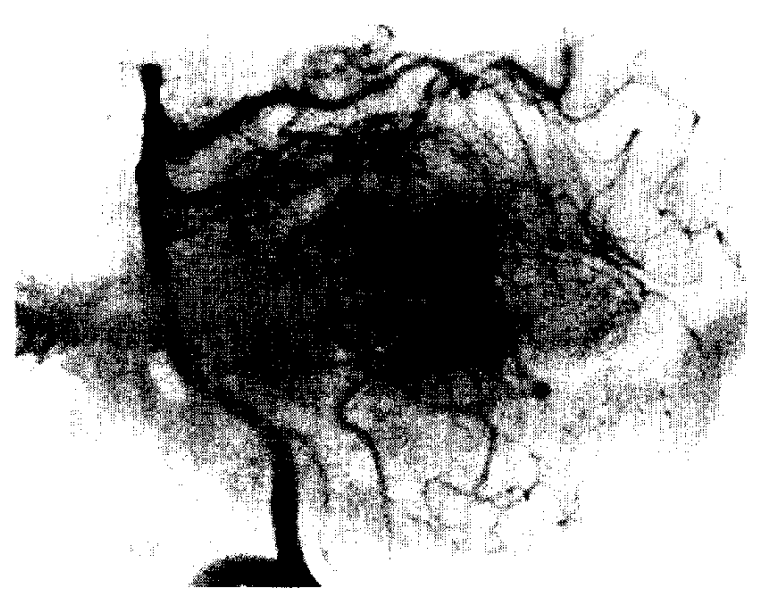

Fig. 4 Left vertebral angiography demonstrating heterogeneous tumor staining. The right posterior inferior cerebellar artery is not visible.

left. The tumor was pinkish gray, soft and elastic, and occupied the right lateral recess of the fourth ventricle. It appeared to have arisen from the inferior tela choroidea. Large feeding arteries branched from the cranial loop of the right posterior inferior cerebellar artery near the choroidal point. The tumor had no dural attachment whatsoever and was completely removed. The histological diagnosis was tran- 


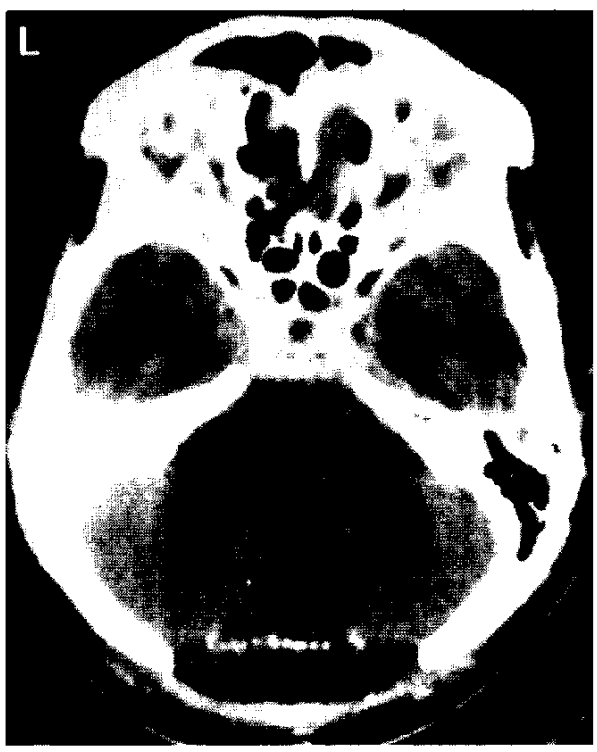

Fig. 5 Postoperative postcontrast CT showing no evidence of residual tumor.

sitional type meningioma. Postoperative CT revealed no residual tumor (Fig. 5). The postoperative course was uneventful and she was discharged 18 days after surgery.

\section{Discussion}

Abraham et al." classified meningiomas of the posterior cranial fossa without dural attachment into three types. Type I meningiomas arise from the choroid plexus of the fourth ventricle and lie entirely within it. Type II meningiomas arise from the inferior tela choroidea and are situated partly in the fourth ventricle and partly in a cerebellar hemisphere. Type III meningiomas are located in the cisterna magna. Our case was a Type II meningioma, which must be distinguished from hypervascular tumors of the fourth ventricle, such as choroid plexus papilloma, ependymoma, and hemangioblastoma.

Meningiomas are easily diagnosed with $\mathrm{CT}$ if they occur in the typical sites. However, correct preoperative diagnosis is more difficult in cases of meningioma arising from the fourth ventricle. Some authors ${ }^{5,6)}$ have emphasized the diagnostic value of vertebral angiography while others ${ }^{2,4,8)}$ have stressed the merits of CT. Cantore et al..$^{21}$ reviewed 22 cases of meningioma of the fourth ventricle and noted the diagnostic usefulness of CT. Although CT demonstrates and localizes tumors of the fourth ventricle, final diagnosis cannot be made on the basis of CT findings alone. We feel that MR imaging is more useful than CT because it provides more precise information about the site, volume, and extent of the lesion. In particular, three-dimensional images clearly delineate the relationships between the tumor and the fourth ventricle and the cerebellar tentorium.

In our case, MR images revealed a homogeneous isointense mass on the $T_{1}$-weighted image, which indicated that it probably was not a hemangioblastoma. Hemangioblastomas are abundantly supplied with blood, and therefore should be represented by low intensity on $T_{1}$-weighted images and signal voids on $\mathrm{T}_{2}$-weighted images. Thus, in our case, the correct preoperative diagnosis was arrived at through MR imaging rather than through CT or vertebral angiography.

\section{References}

1) Abraham J, Chandy J: Meningiomas of the posterior fossa without dural attachment: A case report. $J$ Neurosurg 20: 177-179, 1963

2) Cantore G, Giappetta P, Delfini R, Raco A: Meningiomas of the posterior cranial fossa without dural attachment. Surg Neurol 25: 127-130, 1986

3) Cushing H, Eisenhardt L: Meningiomas: Their Classification, Regional Behavior, Life History and Surgical End Results. Springfield, CC Thomas, 1938, pp 139-140

4) Gökalp HZ, Ozkal E, Erdogan A, Selcuki M; A giant meningioma of the fourth ventricle associated with Stürge-Weber disease. Acta Neurochir (Wien) 57: 115-120, 1981

5) Hoffman JC, Bufkin WJ, Richardson HD: Primary intraventricular meningiomas of the fourth ventricle. Amer J Roentgen 115: 100-104, 1972

6) Rodriguez-Carbajal J, Palacious E: Intraventricular meningiomas of the fourth ventricle. Amer J Roentgen 120: 27-31, 1974

7) Schaerer JP, Woolsey RD: Intraventricular meningiomas of the fourth ventricle. $J$ Neurosurg 17: $337-341,1960$

8) Tsuboi K, Nose T, Maki Y: Meningioma of the fourth ventricle: A case report. Neurosurgery 13: $163-166,1983$

9) Vogel FS, Stevenson LD: Meningothelial meningioma of the fourth ventricle. $J$ Neuropath Exp Neurol 9: 443-448, 1950

Address reprint requests to: S. Nakano, M.D., Department of Neurosurgery, Miyazaki Medical College, 5200 Kihara, Kiyotake-cho, Miyazaki-gun, Miyazaki 889-16, Japan. 